Arch. Tierz., Dummerstorf 51 (2008) 5, 458-466

Fachgebiet Tierhaltung und Haltungsbiologie, Institut für Tierzucht und Haustiergenetik, Justus-Liebig-Universität Gießen, Deutschland

ANNE ELKMANN und STEFFEN HOY

\title{
Untersuchungen zum Beschäftigungsverhalten von weiblichen und kastrierten männlichen Mastschweinen
}

\begin{abstract}
Title of the paper: Investigation on occupational behaviour of female and castrated male fattening pigs In four rounds 144 fattening pigs of both sexes were brought in four pens (two of them were littered, two without any litter). Their occupational behaviour was recorded at the time of bringing the pigs into the pen, at the beginning, at the middle and at the end of the fattening period using an infrared-video-technology and a time lapse video recorder. In every pen three occupational tools (pendular beam, cross of chains and lifting beam) were offered to the pigs. At the $1^{\text {st }}$ recording the behaviour of all pigs was analysed, at the other fattening periods the behaviour of six focal pigs of both sexes per pen was analysed always over $24 \mathrm{~h}$. At the time the pigs were brought into the pens no significant differences between the two sexes could be observed concerning the latency until the pigs had the first contact to the different occupational tools, whereas both sexes needed longest for their first contact to the cross of chains. During the other fattening periods the duration of occupation with the cross of chains $(\hat{\delta}=0.06 \% ;$ q $=0.08 \%, \mathrm{p}<0.086)$ and the pendular beam $(\hat{\delta}=0.15 \% ; \phi=0.2 \%, \mathrm{p}<0.087)$ by the female pigs tended to be longer than the one shown by the castrated male pigs. Thereby both sexes used the cross of chains longer for occupation than the pendular beam and the lifting beam. The female pigs occupied themselves significantly longer with the straw in the littered pens, the floor in the pens without straw, the pen's equipment and the pen mates than the castrated male pigs did. In contrast the castrated males used the trough significantly longer for occupation. In this study female pigs were more active, whereas the castrated male pigs spent a significantly longer time feeding and tended to spend more time lying and had better daily gains.
\end{abstract}

Keywords: fattening pigs, sex, occupational tools, occupational behaviour

\section{Zusammenfassung}

In vier Durchgängen wurden 144 Mastschweine in vier Buchten (zwei eingestreut, zwei einstreulos) Geschlechter gemischt aufgestallt und deren Beschäftigungsverhalten mittels Infrarot-Videotechnik und Langzeitvideorecorder bei der Einstallung, zu Mastanfang, Mastmitte und Mastende aufgezeichnet. In jeder Bucht wurden den Tieren drei Beschäftigungsgeräte (Pendelbalken, Kettenkreuz und Hebebalken) angeboten. Bei der Einstallung wurde das Verhalten aller Tiere analysiert, bei den anderen Mastzeitpunkten erfolgte eine Analyse des Verhaltens von je sechs Fokustieren beiderlei Geschlechts pro Bucht immer über $24 \mathrm{~h}$. Bei der der Einstallung zeigten sich in der Zeitdauer bis zum ersten Kontakt mit den einzelnen Geräten keine signifikanten Unterschiede zwischen den Geschlechtern, wobei beide Geschlechter beim Kettenkreuz am längsten brauchten, bis sie sich das erste Mal damit beschäftigten. Im weiteren Mastverlauf war die Dauer bei der Beschäftigung mit dem Kettenkreuz $(\hat{\delta}=0,06 \%$; $,=0,08 \%, \mathrm{p}<0,086)$ und dem Pendelbalken $(\hat{\delta}=0,15 \%$; $q=0,2 \%, p<0,087)$ bei den weiblichen Tieren tendenziell länger als bei den Kastraten. Dabei nutzten beide Geschlechter das Kettenkreuz länger als den Pendelbalken und den Hebebalken. Mit dem Stroh in den eingestreuten Buchten, mit dem Boden in den einstreulosen Buchten, mit der Buchteneinrichtung sowie mit dem Buchtenpartner beschäftigten sich die weiblichen Tiere signifikant länger als die Kastraten. Diese nutzten dagegen den Trog signifikant länger zur Beschäftigung. Weibliche Schweine zeigten sich in dieser Studie aktiver als Kastraten, wobei diese signifikant mehr Zeit mit dem Fressen und tendenziell mehr Zeit liegend verbrachten und höhere tägliche Zunahmen erzielten. 


\section{Einleitung}

In Deutschland findet die Haltung von Schweinen, insbesondere auch die Haltung von Mastschweinen zu 88\% einstreulos statt (KANSWOHL, 2000; KESSEN, 2005). Die Tiere werden zumeist in Teil- oder Vollspaltenbodenbuchten gehalten, die durch ihre einfache Ausführung den Schweinen nur wenige Möglichkeiten zur Erkundung und Beschäftigung bieten. Schweine verbringen jedoch sehr viel Zeit des Tages mit der Nahrungssuche und der Erkundung ihrer Umwelt, sofern ihnen ein entsprechender Anreiz geboten wird. Fehlen den Tieren Erkundungsmöglichkeiten, so können Verhaltensstörungen, wie Schwanz- oder Ohrenbeißen, die Folge sein (BLACKSHAW, 1981; STOLBA und WOOD-GUSH, 1981; WEBER und VALLE ZARATE, 2005). Der Gesetzgeber fordert das ständige Anbieten von ausreichenden Mengen an zu untersuchenden und veränderbaren Materialien, welche für das Tier gesundheitlich unbedenklich sind. Im Gegensatz zur EU-Richtlinie 2001/93/EG werden dabei in der Tierschutz-Nutztierhaltungsverordnung aber keine Materialen wie Stroh, Heu, Holz oder Torf vorgegeben. Es stellt sich zum einen die Frage, welche Beschäftigungsgeräte geeignet sind. Zum anderen ist unklar, ob es Unterschiede bei der Beschäftigungsdauer zwischen den Geschlechtern gibt. Bezüglich des geeigneten Beschäftigungsmaterials zeigen viele Studien, dass Stroh oder auch erdartige Materialien wie Torf den Tieren gute Erkundungsmöglichkeiten bieten und auch gut angenommen werden (FRASER et al., 1991; SAMBRAUS, 1997; BEATTIE et al. 2000a, 2000b, 2001; ROHRMANN und HOY, 2004). Jedoch muss auf eventuelle hygienische Probleme auf Grund von Mykotoxinen oder auch Mykobakterien hingewiesen werden. Auch Schwierigkeiten mit dem Güllemanagement sowie ökonomische Gründe sollten nicht außer Acht gelassen werden. Beschäftigungsgeräte aus Ketten, Seilen, Holz aber auch Autoreifen oder Kanistern werden in vielen landwirtschaftlichen Betrieben angeboten. Dabei geben sowohl ELLERSIEK (1994) als auch HEGGEMANN (2001) an, dass die Beschäftigungsgeräte aus hygienischen Gründen nicht auf dem Boden liegen sollten. Die Geräte oder Materialien sollen immer frei hängen bzw. über spezielle Halterungen angeboten werden. Ziel dieser Arbeit war es, die Nutzung unterschiedlicher Geräte (Kettenkreuz, Hebebalken, Pendelbalken) bei einstreuloser oder eingestreuter Haltung unter besonderer Berücksichtigung des Geschlechtes zu untersuchen.

Tiere, Material und Methoden

Die Daten wurden in vier Mastdurchgängen an insgesamt 144 Tieren (67 Kastraten und 77 weibliche Mastschweine) der Genetik Westhybrid $\times$ Piétrain erfasst. Je Mastdurchgang wurden 36 Tiere in vier Untersuchungsbuchten $\left(8,55 \mathrm{~m}^{2}=0,95 \mathrm{~m}^{2}\right.$ pro Tier) eingestallt, von denen zwei eingestreut und zwei einstreulos (Teilspaltenboden) waren. In zwei Durchgängen hatten die Tiere $14 \mathrm{~h}$ und in zwei weiteren Durchgängen $8 \mathrm{~h}$ Licht am Tag zur Verfügung, welches über eine Zeitschaltuhr gesteuert wurde. Die Tiere wurden nach Geschlechtern gemischt in möglichst ausbalanciertem Verhältnis in die Buchten eingestallt. Bei allen Tieren waren die Schwänze kupiert. Das Einstallgewicht der Tiere lag durchschnittlich bei $33 \mathrm{~kg}$, das Ausstallgewicht bei durchschnittlich $119 \mathrm{~kg}$. Die Tiere wurden im Mastverlauf regelmäßig gewogen. Die letzte gemeinsame Wägung der Tiere erfolgte nach durchschnittlich 81 Tagen Mastdauer zu „Mastende“. Die Ausstallung der Tiere erfolgte dann phasenweise, um ein für den Schlachthof gefordertes optimales Gewicht zu erreichen. 
Die Fütterung der Tiere erfolgte ad libitum über in die Zwischenwand eingebaute Breiautomaten. Für eine Ad libitum-Wasseraufnahme stand den Tieren zusätzlich zu den beiden Sprühnippeln im Breiautomaten eine Nippeltränke pro Bucht zur Verfügung.

In jede Bucht wurden drei selbstgebaute Beschäftigungsgeräte, ein Pendelbalken, ein Kettenkreuz und ein Hebebalken installiert. Der Pendelbalken bestand aus einem 51 $\mathrm{cm}$ langen und $4,5 \mathrm{~cm}$ dicken Stück Holz, welches über eine Kette an der Buchtendecke befestigt wurde. Das Kettenkreuz bestand aus zwei $51 \mathrm{~cm}$ langen kreuzförmig verschweißten Metallstäben, welche ebenfalls über eine Kette an der Buchtendecke befestigt war. An beiden Enden der Metallstäbe wurden $44 \mathrm{~cm}$ lange Ketten mit daran fixierten Holzstücken (14,5 cm lang, 4,5 cm dick) angebracht. Der Hebebalken bestand aus einem etwa $30 \mathrm{~cm}$ langen Holzstück, welches über ein $18 \mathrm{~cm}$ langes Flacheisen an einem Metallrohr mit einem Durchmesser von 3,5 cm befestigt war. Dieses verlief in einem weiteren Metallrohr von $4 \mathrm{~cm}$ Durchmesser, in welchem im oberen Abschnitt seitlich zwei Kerben angebracht waren, durch die das innere Rohr über eine Schraube fixiert werden konnte (ELKMANN, 2008).

Bei der Einstallung der Tiere erfolgte für die Analyse der Latenzzeit bis zum ersten Kontakt mit den drei installierten Beschäftigungsgeräten eine 24stündige Aufzeichnung des Verhaltens der Tiere. Zu Mastanfang, zu Mastmitte und zu Mastende fanden weitere Videoaufzeichnungen für die Verhaltensuntersuchung über drei Tage mittels Infrarot-Videotechnik und Langzeitvideorecorder immer über $24 \mathrm{~h}$ statt. Am Abend vor dem Start einer Videoaufzeichnung wurden die Strohbuchten ausgemistet und neu eingestreut. Um das Verhalten von Einzeltieren später analysieren zu können, wurden in jeder Bucht zu diesen Zeitpunkten jeweils sechs Fokustiere mittels eines Punkt-Strich-Systems markiert. Die Auswahl der Fokustiere erfolgte zu Mastanfang zufällig, zu den beiden anderen Maststadien wurden dann dieselben Tiere wie zu Mastanfang markiert. Dabei wurden möglichst drei Kastrate und drei weibliche Tiere pro Bucht ausgewählt. Die Analyse der aufgezeichneten Videos erfolgte mit Hilfe des Programms „THE OBSERVER ${ }^{\circledR}$ “ (Noldus). Dadurch konnte das Verhalten der Tiere anhand von 17 erfassten Verhaltensparametern (Tabelle 1) sekundengenau immer über $24 \mathrm{~h}$ ausgewertet werden.

Die erste statistische Bearbeitung der Daten erfolgte durch die deskriptive Statistik des Observers-Programmes. Die Daten wurden dann sowohl auf Basis der prozentualen Beschäftigung in $24 \mathrm{~h}$ als auch auf Basis der absoluten Zeitdauer in $\mathrm{s}$ zusammengefasst und mittels des Statistikprogramms SPSS for Windows weiter bearbeitet. Nach einem Test auf Normalverteilung zeigte sich, dass einige der Verhaltensparameter nicht normalverteilt waren. Für die Daten der Zeitdauer bis zum ersten Kontakt mit den Beschäftigungsgeräten konnte über eine Logarhythmusfunktion eine annähernde Normalverteilung erreicht werden, so dass eine univariate Varianzanalyse mit folgendem Modell berechnet wurde:

$$
y_{i j}=\mu+\text { Haltungssystem }_{i}+\text { Geschlecht }_{j}+e_{i j}
$$

wobei $y$ die Zeitdauer bis zum ersten Kontakt mit den Beschäftigungsgeräten ist.

Bei den Verhaltensdaten mit Auswertung über den Observer wurde bei den nicht normalverteilten Daten geprüft, ob sich über eine Wurzeltransformation eine Normalverteilung erreichen ließ. Bei allen dann annähernd normalverteilten Daten erfolgte eine Berechnung mittels univariater Varianzanalyse nach folgendem Modell: 


$$
\begin{aligned}
& y_{i j k l}=\mu+\text { Haltungssystem }_{i}+\text { Maststadium }_{j}+\text { Licht }_{k}+\text { Geschlecht }_{l} \\
& +(\text { signifikant getestete Interaktionen für die jeweilige Verhaltensweise })+e_{i j k l}
\end{aligned}
$$

wobei $y$ liegen, laufen, stehen, sitzen, Beschäftigung mit dem Trog, der Tränke, den Beschäftigungsgeräten, der Buchteneinrichtung, dem Buchtenpartner u. a. ist.

Tabelle 1

Erfasste Verhaltensmerkmale (Behaviour traits)

\begin{tabular}{ll}
\hline Verhaltensmerkmale & Beschreibung \\
\hline laufen & laufen \\
liegen & liegen in Seiten- oder Brustlage \\
Pendelbalken & Beschäftigung mit dem Pendelbalken \\
Kettenkreuz & Beschäftigung mit dem Kettenkreuz \\
Hebebalken & Beschäftigung mit dem Hebebalken \\
Stroh & Beschäftigung mit Stroh (in der Strohbucht) \\
Boden & Beschäftigung mit dem Boden (in der Spaltenbodenbucht) \\
stehen & stehen \\
sitzen & sitzen \\
Trog & Beschäftigung mit dem Trog, wobei hier der Aufenthalt am Trog (Kopf im Trog und \\
& Bewegen der Dosiereinrichtung) erfasst wurde. Es konnte nicht unterschieden werden, \\
ob das Tier frisst, wühlt, trinkt oder sich spielerisch mit dem Futter beschäftigte. & trinken \\
Tränke & Beschäftigung mit Buchteneinrichtungen, der Wand, dem Tor, dem Trogbereich \\
Buchteneinrichtung & oberhalb der Futterglocke oder daneben \\
Schwanz & Beschäftigung mit dem Schwanz eines Buchtenpartners \\
Ohr & Beschäftigung mit dem Ohr eines Buchtenpartners \\
Buchtenpartner & Beschäftigung mit dem Buchtenpartner; dazu gehörten z. B. das Verdrängen vom Trog, \\
aufreiten & Lecken, Massieren, Wegjagen in der Bucht \\
scheuern & aufreiten auf den Buchtenpartner \\
Gesamtaktivität & Zcheuern an der Wand oder anderen Buchtengegenständen \\
& Zusammenfassung aller Verhaltensparameter ohne Liegen
\end{tabular}

Bei Verhaltensparametern, die sich auch durch eine Wurzeltransformation nicht normalverteilen ließen, erfolgte die weitere statistische Bearbeitung über nichtparametrische Tests, wie den Mann-Whitney-U-Test oder den Kruskal-Wallis-H-Test. Neben den Verhaltensdaten wurde auch die Lebendmasseentwicklung der Tiere in regelmäßigen Abständen (Einstallung; Mastmitte; Mastende; Ausstallung) erfasst. Die statistische Bearbeitung dieser Daten erfolgte ebenfalls über univariate Varianzanalysen nach folgendem Modell:

$$
\begin{aligned}
& y_{i j k l}=\mu+\text { Haltungssystem }_{i}+\text { Licht }_{j}+\text { Geschlecht }_{k}+b\left(\text { Einstallgewich }_{l}\right. \\
& - \text { Einstallgewicht }_{l}+e_{i j k l}
\end{aligned}
$$

wobei $y$ die tägliche Zunahme und Gesamtzunahme ist.

\section{Ergebnisse}

Die Analyse der Videoaufzeichnung bei der Einstallung zeigte, dass sowohl die weiblichen Tiere als auch die Kastraten beim Kettenkreuz mit 5,7 min am längsten brauchten, bis sie den ersten Kontakt zu diesem Gerät hatten. Am schnellsten erkundeten die männlichen Tiere den Hebebalken $(3,2 \mathrm{~min})$, bei den weiblichen Tieren war es dagegen der Pendelbalken (3,8 min) mit dem schnellsten Erstkontakt. Die Unterschiede in der Zeitdauer bis zum ersten Kontakt mit dem jeweiligen Gerät erwiesen sich zwischen den beiden Geschlechtern jedoch als nicht signifikant. Bei der Beschäftigungsdauer während dieses ersten Kontaktes gab es bei beiden 
Geschlechtern höchstsignifikante Unterschiede zwischen den Geräten, jedoch unterschied sich die Beschäftigungsdauer bei den einzelnen Geräten zwischen den Geschlechtern nicht signifikant. So nutzten sowohl die Kastraten mit 6,8 s als auch die weiblichen Tiere mit 8,1 s den Hebebalken am längsten beim ersten Kontakt. Das Kettenkreuz wurde von beiden Geschlechtern mit 3 s gleichlang beim ersten Kontakt genutzt. Der Pendelbalken wurde von den Kastraten 2,6 s und von den weiblichen Tieren $4 \mathrm{~s}$ erkundet (Abbildung 1).

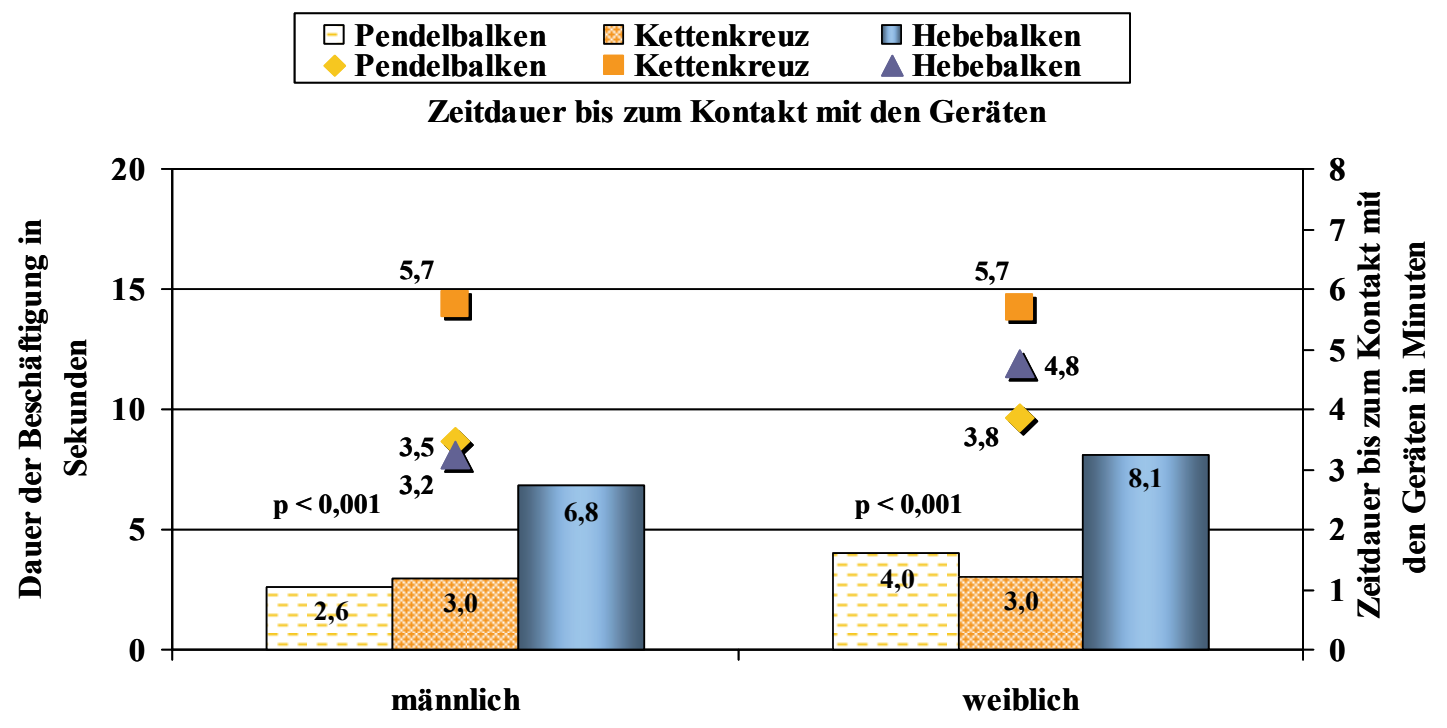

Dauer der ersten Beschäftigung

Abb. 1: Zeitdauer bis zum ersten Kontakt mit den Beschäftigungsgeräten (Punkte) und Dauer der ersten Beschäftigung bei den unterschiedlichen Geschlechtern (Säulen) (Duration of time until the first contact with the occupational tools [points] and the duration of the first occupation by the different sexes [pillars])

Bei der Beschäftigung mit den Geräten während der Mast konnten keine signifikanten Unterschiede zwischen den Kastraten und den weiblichen Fokustieren festgestellt werden (Tabelle 2). Jedoch deutete sich an, dass sich die weiblichen Tiere pro Tag tendenziell mehr mit dem Pendelbalken $(\hat{\delta}=0,06 \% ;$; $=0,08 \%, p<0,086)$ und dem Kettenkreuz $(\hat{\delta}=0,15 \%$; $q=0,2 \%, p<0,087)$ beschäftigten als die Kastraten. Dabei wurde das Kettenkreuz sowohl bei den Kastraten als auch bei den weiblichen Fokustieren gegenüber den anderen beiden Beschäftigungsgeräten bevorzugt. Bei der Beschäftigung mit dem Stroh in den Strohbuchten sowie bei der Beschäftigung mit dem Boden in den einstreulosen Haltungssystemen waren die weiblichen Fokustiere signifikant aktiver als die Kastraten. So beschäftigten sich die weiblichen Tiere zu 4,94\% in $24 \mathrm{~h}$ mit dem Stroh in den Strohbuchten, die Kastraten mit 3,81\% in $24 \mathrm{~h}$ hochsignifikant weniger. Die Beschäftigung mit dem Boden in den einstreulosen Haltungssystemen lag bei beiden Geschlechtern unter der Beschäftigung mit dem Stroh in den eingestreuten Buchten, jedoch waren auch hier die weiblichen Fokustiere mit einer Beschäftigungsdauer von $3,42 \%$ in $24 \mathrm{~h}$ signifikant aktiver als die Kastraten $(2,75 \%$ in $24 \mathrm{~h} ; \mathrm{p}<0,05)$. Auch bei der täglichen Beschäftigung mit der Buchteneinrichtung $(\hat{\delta}=0,67 \%$; $q=0,82 \%, \mathrm{p}<0,05)$ und dem Buchtenpartner $(\hat{\delta}=$ $0,77 \%$; $q=0,92 \%, \mathrm{p}<0,05)$ zeigten sich die weiblichen Fokustiere aktiver als die Kastraten. Außerdem verbrachten sie mit $1,38 \%$ in $24 \mathrm{~h}$ eine längere Zeit mit Laufen als die Kastraten $(1,19 \%$ in $24 \mathrm{~h}, \mathrm{p}<0,01)$. Die Kastraten beschäftigten sich dagegen täglich länger mit dem $\operatorname{Trog}(\hat{\sigma}=8,79 \% ; q=8,06 \%, p<0,01)$. Bei den anderen 
analysierten Verhaltensparametern konnten dagegen keine signifikanten Unterschiede zwischen den Geschlechtern beobachtet werden. Doch wie die Gesamtaktivität zeigt, waren die weiblichen Fokustiere im Vergleich zu den Kastraten tendenziell länger aktiv $(\hat{\delta}=19,02 \% ;$; $=19,91 \%, \mathrm{p}<0,061)$. Die Kastraten verbrachten dagegen tendenziell pro Tag mehr Zeit mit Liegen, waren also inaktiver $(\hat{\delta}=80,98 \%$; $+=80,09 \%, p<0,061)$.

Tabelle 2

Beschäftigung der Kastraten und der weiblichen Fokustiere in 24 h (Ergebnisse der univariaten Varianzanalyse sowie der nichtparametrischen Tests) (Occupational behaviour of the female and castrated male focal pigs in $24 \mathrm{~h}$ [Results of univariate analysis of variance as well as the results of the nonparametric tests])

\begin{tabular}{|c|c|c|c|}
\hline Verhaltensmerkmale & $\mathrm{n}=141 \cdot 24 \mathrm{~h}$ & $q \mathrm{n}=147 \cdot 24 \mathrm{~h}$ & $\mathrm{p}$ \\
\hline Pendelbalken $^{1)}$ & 0,06 & 0,08 & n.s. $(p<0,086)$ \\
\hline Kettenkreuz ${ }^{1)}$ & 0,15 & 0,20 & n.s. $(\mathrm{p}<0,087)$ \\
\hline Hebebalken $^{1)}$ & 0,11 & 0,10 & n.s \\
\hline Beschäftigung gesamt mit allen drei Geräten ${ }^{1)}$ & 0,39 & 0,46 & n.s. \\
\hline Stroh* & 3,81 & 4,94 & 0,01 \\
\hline Boden* & 2,75 & 3,42 & 0,05 \\
\hline Buchteneinrichtung & 0,67 & 0,82 & 0,05 \\
\hline Buchtenpartner ${ }^{1)}$ & 0,77 & 0,92 & 0,05 \\
\hline $\mathrm{Ohr}^{1)}$ & 0,12 & 0,15 & n.s. \\
\hline Schwanz $^{2)}$ & 0,003 & 0,003 & n.s. \\
\hline Trog & 8,79 & 8,06 & 0,01 \\
\hline Tränke ${ }^{1)}$ & 0,3 & 0,28 & n.s. \\
\hline Laufen & 1,19 & 1,38 & 0,01 \\
\hline Stehen & 1,59 & 1,53 & n.s. \\
\hline Sitzen ${ }^{1)}$ & 1,43 & 1,62 & n.s. \\
\hline Gesamtaktivität & 19,02 & 19,91 & n.s. $(p<0,061)$ \\
\hline Liegen & 80,98 & 80,09 & n.s. $(p<0,061)$ \\
\hline
\end{tabular}

* Daten bei der Berechnung nach Haltungssystem geteilt; univariate Varianzanalyse ohne Faktor Haltungssystem

1) zurücktransformierte LSQ-Mittelwerte; 2) Rohdaten; Statistik mit nichtparametrischen Tests

Die Ergebnisse der Lebendmasseentwicklung zeigten, dass die Kastraten bis zur letzten gemeinsamen Wägung zu „Mastende“ ein höchstsignifikant höheres

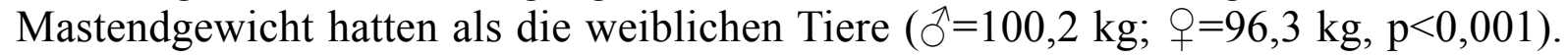
Daraus resultierte bis zu diesem Mastzeitpunkt auch eine höhere Gesamtzunahme $(\hat{\jmath}=67,2 \mathrm{~kg} ; \mathrm{Q}=63,3 \mathrm{~kg}, \mathrm{p}<0,01)$ sowie höhere tägliche Zunahmen $(\hat{\jmath}=831 \mathrm{~g} ; \mathrm{q}=776$ $\mathrm{g}, \mathrm{p}<0,01)$ der Kastraten gegenüber den weiblichen Schweinen. Das mittlere Einstallgewicht betrug dabei bei den Kastraten $33,0 \mathrm{~kg}$ und bei den weiblichen Schweinen 32,96 kg und unterschied sich nicht signifikant voneinander (Tabelle 3).

Tabelle 3

Lebendmasseentwicklung der Kastraten und der weiblichen Tiere (Ergebnisse der univariaten Varianzanalyse) (Development of life weight of castrated male and the female pigs [Results of univariate analysis of variance])

\begin{tabular}{lccl}
\hline & $\mathrm{o}$ & $q$ & $\mathrm{p}$ \\
\hline Mastperiode: Einstallen bis Mastende & & & \\
Einstallgewicht $\mathrm{t}^{1}(\mathrm{~kg})$ & 33,0 & 32,96 & n.s. \\
Mastendgewicht $(\mathrm{kg})$ & 100,2 & 96,3 & $\mathrm{p}<0,001$ \\
Gesamtzunahme $(\mathrm{kg})$ & 67,2 & 63,3 & $\mathrm{p}<0,01$ \\
tägliche Zunahme $(\mathrm{kg})$ & 0,831 & 0,776 & $\mathrm{p}<0,01$ \\
Masttage bis Mastende & 81,1 & 81,7 & n.s. \\
\hline
\end{tabular}

1) Statistik mit dem t-Test (Einstallgewicht: Kovariable in der Varianzanalyse), 2) Rohdaten; Statistik mit nichtparametrischen Tests 


\section{Diskussion}

Es zeigt sich, dass sich die Beschäftigung von weiblichen Fokustieren und Kastraten innerhalb der 24stündigen Beobachtungszeit unterschied. Es gab zwar keine signifikanten Unterschiede zwischen den beiden Geschlechtern hinsichtlich der Beschäftigung mit den angebotenen Geräten Pendelbalken, Kettenkreuz oder Hebebalken, doch beschäftigten sich weibliche Tiere zumindest tendenziell länger mit dem Kettenkreuz und dem Pendelbalken als die Kastraten. Dabei lag die Dauer der Beschäftigung mit dem Kettenkreuz bei beiden Geschlechtern über der mit dem Hebebalken und dem Pendelbalken. Beide Geschlechter bevorzugten das Kettenkreuz, was in der Bauweise des Gerätes begründet sein kann. Durch die freie Aufhängung schwingen alle Ketten mit, wenn sich ein Tier mit einer Kette oder dem daran befestigten Holzstück beschäftigt. Dadurch gab es für andere Tiere sowohl visuell als auch akustisch durch das Rasseln der Ketten einen Anreiz zur Mitbeschäftigung.

Außerdem bot das Kettenkreuz durch die vier Ketten gleich mehreren Tieren eine Möglichkeit zum Spielen. ELLERSIEK (1994) berichtet ebenfalls von dem zusätzlichen akustischen Lockeffekt, der durch das Kettenrasseln entsteht. BLACKSHAW et al. (1997) konnten feststellen, dass Schweine ein fixiert angebotenes Beschäftigungsmaterial besser annahmen als eines, das auf dem Boden lag. Beim ebenfalls frei schwingenden Pendelbalken waren die visuellen und akustischen Reize offensichtlich nicht so stark ausgeprägt, so dass sich sowohl die Kastraten als auch die weiblichen Tiere weniger damit beschäftigten. Der Hebebalken war durch seine festere Fixierung gut für die Tiere zu benagen, und sie konnten ihn anheben, was dieses Gerät für beide Geschlechter interessanter machte als der Pendelbalken.

Die Dauer der Beschäftigung mit dem Stroh in den Strohbuchten war bei den weiblichen Fokustieren hochsignifikant länger als die der Kastraten. Auch bei der Beschäftigung mit dem Boden in den Spaltenbodenbuchten, der Beschäftigung mit der Buchteneinrichtung und dem Buchtenpartner sowie bei der Zeit, in der die Tiere liefen, waren die weiblichen Tiere signifikant aktiver als die Kastraten. Diese beschäftigten sich dagegen signifikant länger mit dem Trog und damit auch länger mit der Futteraufnahme, was die signifikant bessere tägliche Zunahme der Kastraten bis $\mathrm{zu}$ Mastende erklärt. Eine bessere Lebendmasseentwicklung von Kastraten fanden auch FUCHS (1992), HYUN et al. (1997) und FISCHER et al. (2006) u.a.

In der vorliegenden Untersuchung zeigte auch die tendenziell längere Gesamtaktivität, dass weibliche Fokustiere aktiver waren als die Kastraten, diese verbrachten dabei eine tendenziell längere Zeitdauer mit dem Liegen. Dieses könnte auch eine Erklärung für die Gewichtsdifferenz der beiden Geschlechter zu Mastende sein. Auch PORZIG und LIEBENBERG (1977) konnten bezüglich der Liegedauer eine geringgradige Differenz zwischen den Geschlechtern der Schweine ausmachen. Die Autoren beobachteten, dass männliche Tiere bei einer Lebendmasse von 21 bis $120 \mathrm{~kg} \mathrm{zu} \mathrm{80,3 \%}$ in $24 \mathrm{~h}$ lagen, weibliche Tiere dagegen zu 79,9\%. Sowohl bezüglich der Beschäftigung mit dem Schwanz als auch bei der Beschäftigung mit den Ohren der Buchtenpartner zeigten sich in der vorliegenden Untersuchung keine Unterschiede zwischen den Geschlechtern. Im Gegensatz dazu konnten PENNY et al. (1972), PENNY und HILL (1974) sowie KRITAS und MORRISON (2004) beobachten, dass männliche Tiere signifikant häufiger bebissen wurden als die weiblichen Tiere. Die Autoren konnten bei Börgen doppelt so viele Verletzungen nachweisen wie bei den weiblichen Schweinen. Auch in Untersuchungen von 
HUNTER et al. (1999) an kupierten und unkupierten Schlachtschweinen wiesen weibliche Schweine $(3,4 \%)$ weniger Verletzungen durch Schwanzbeißen auf als die Börge (4,6\%).

Um der Forderung der EU-Richtlinie 2001/93/EG bzw. der Tierschutz-Nutztierhaltungsverordnung nachzukommen, ist es zwingend notwendig, Schweinen Beschäftigungsmaterial anzubieten. Diese Untersuchung konnte zeigen, dass auch Beschäftigungsgeräte ohne Stroh sowohl von Kastraten als auch von weiblichen Schweinen zur Beschäftigung genutzt werden. Jedoch werden Geräte, die mehreren Tieren gleichzeitig eine Beschäftigung bieten, von beiden Geschlechtern bevorzugt. Gerade in großen Mastgruppen ist es daher empfehlenswert, den Tieren mehrere unterschiedliche Beschäftigungsgeräte anzubieten, um zum einen mehreren Tieren gleichzeitig eine Beschäftigung $\mathrm{zu}$ ermöglichen und zum anderen für mehr Abwechslung zu sorgen. Die Tiere können so ganz unterschiedliche Verhaltensweisen, wie Ziehen, Zerren, Schütteln, Kauen, Heben und Wühlbewegungen, ausüben. Wird Holz als Beschäftigungsmaterial gewählt, sollten die Holzstücke aus hygienischen Gründen nach jedem Mastdurchgang gewechselt werden.

\section{Literatur}

BEATTIE, V.E.; O'CONNEL, N.E.; KILPATRICK, D.J.; MOSS, B.W.: Influence of environmental enrichment on behaviour, performance and meat quality of domestic pigs. Livest. Prod. Sci. 65 (2000a), 71-79

BEATTIE, V.E.; O'CONNEL, N.E.; KILPATRICK, D.J.; MOSS, B.W.: Influence of environmental enrichment on welfare-related behavioural and physiological parameters in growing pigs. Anim. Sci. 70 (2000b), 443-450

BEATTIE, V.E.; SNEDDON, I.A.; WALKER, N.; WEATHERUP, R.N.: Environmental enrichment of intensive pig housing using spent mushroom compost. Anim. Sci. 72 (2001), S. 35-42

BLACKSHAW, J.K.: Some behavioural deviations in weaned domestic pigs: persistent inguinal nose thrusting, and tail and ear biting. Anim. Prod. 33 (1981), 325-332

BLACKSHAW, J.K.; THOMAS, F.J.; LEE, J.A.:

The effect of a fixed or free toy on the growth rate and aggressive behaviour of weaned pigs and the influence of hierarchy on initial investigation on the toys. Appl. Anim. Behav. Sci. 53 (1997), 203-212

ELKMANN, A.:

Haltungsbiologische Untersuchungen zur Beschäftigung von Mastschweinen in einstreuloser oder eingestreuter Haltung. Dissertation Universität Giessen (2008)

ELLERSIEK, H. H. : Ketten, Bälle, Reifen oder was? Top agrar 3 (1994), S1

FISCHER, K.; LINDNER; J.P.; JUDAS, M.; HÖRETH, R.:

Schlachtkörperzusammensetzung und Gewebebeschaffenheit von schweren Schweinen. 1. Mitteilung: Material und Methoden, Mastleistung, Schlachtkörperzusammensetzung und Teilstückanteile. Arch. Tierz. 49 (2006), 269-278

FRASER, D.; PHILLIPS, P.A.; THOMPSON, B.K.; TENNESSEN, T.:

FUCHS, C.: Effect of straw on the behaviour of growing pigs. Appl. Anim. Behav. Sci. 30 (1991), 307-318

HEGGEMANN, R.: So Schwanzbeißen vermeiden. dlz agrarmagazin 4 (2001), 132-137

HUNTER, E.J.; JONES, T.A.; GUISE, H.J.; PENNY, R.H.C.; HOSTE, S.:

Tail biting in pigs 1: the prevalence at six UK abattoirs and the relationship of tail biting with docking, sex and other carcass damage. Pig. J. 43 (1999), 18-32

HYUN, Y.; ELLIS, M.; MCKEITH, K.; WILSON, E.R.:

Feed intake pattern of group-housed growing-finishing pigs monitored using a computerized feed intake recording system. J. Anim. Sci. 75 (1997), 1443-1451 


\title{
KANSWOHL, N.:
}

Haltungsverfahren unter Berücksichtigung von Tiergerechtigkeit, Umweltschutz und rationeller KESSEN, R.: Produktion. Arch. Tierz. 43 (2000) Special Issue, 21-27

aid-infodienst: presse-info-achiv. Ausgabe Nr.45/05 vom 10.11.05, Stallhaltungsverfahren (2005) http://www.aid.de/presse/presseinfo_archiv.php? mode=beitrag\&id=2244

KRITAS, S.K.; MORRISON, R.B.:

An observational study on tail biting in commercial grower-finisher barns. J. Swine Health Prod. 12 (2004), 17-22

PENNY, R.H.C.; HILL, F.W.G.; FIELD, J.E.; PLUSH, J.T.:

Tail-biting in pigs: a possible sex incidence. The Veterinary Record 91 (1972), 482

PENNY, R.H.C.; HILL, F.W.G.:

Observations of some conditions in pigs at the abattoir with particular reference to tail biting. The Veterinary Record 94 (1974), 174-180

PORZIG, E.; LIEBENBERG, O.:

Untersuchungen zum Verhalten von Mastschweinen unter besonderer Berücksichtigung der Ontogenese von Verhaltensweisen. 1. Mitteilung: Zur Entwicklung des Liegeverhaltens und des Sitzens. Arch. Tierz. 20 (1977), 107-117

ROHRMANN, S.; HOY, S.:

Ethologische Untersuchungen zur Beschäftigung von Ebern in Besamungsstationen mit Stroh. Arch. Tierz. 47 (2004), 565-574

SAMBRAUS, H.H.:

Tierverhalten - Anzeiger für eine artgerechte Tierhaltung. Arch. Tierz. 40 (1997) Sonderheft, 26-34

STOLBA, A.; WOOD-GUSH, D.G.M.:

Verhaltensgliederung und Reaktion auf Neureize als ethologische Kriterien zur Beurteilung von Haltungsbedingungen bei Hausschweinen. In: Aktuelle Arbeiten zur artgemäßen Tierhaltung 1980. KTBL-Schrift 264 (1981), 110-128

WEBER, R.E.F.; VALLE ZARATE, A.:

Der Begriff Wohlbefinden in der Nutztierhaltung - Diskussion aktueller Definitionsansätze als Grundlage für praxisorientierte Forschung am Beispiel Mastschweinehaltung. Arch. Tierz. 48 (2005), 475-489

Eingegangen: 2008-04-01

Akzeptiert: 2008-07-07

\author{
Autoren: \\ Dr. ANNE ELKMANN* \\ Prof. Dr. STEFFEN HOY \\ Institut für Tierzucht und Haustiergenetik \\ Justus-Liebig-Universität Gießen \\ Bismarckstraße 16 \\ D-35390 Gießen \\ Deutschland \\ *korrespondierender Autor \\ E-Mail: a.elkmann@gmx.de
}

